\title{
Radiology and patient communication: if not now, then when?
}

\author{
Julie Cox ${ }^{1,2}$ - Yitka Graham ${ }^{1,2}$
}

Received: 6 May 2019 /Revised: 10 June 2019 / Accepted: 28 June 2019/Published online: 29 July 2019

(C) European Society of Radiology 2019

\section{Key Points}

- Communication with patients in radiology is, in general, indirect using the referrer as a conduit.

- Direct patient communication may be beneficial for radiology departments and radiologists to improve patient awareness about the nature of our role and also to provide correct and measured information about the nature and frequency of discrepancies in radiology.

Communication with patients, a cornerstone of medical practice globally, is anomalous in the specialty of diagnostic radiology in that communication is generally indirect. Radiologists receive imaging requests from referring clinicians and send written examination reports to the referrers, which excludes patients from direct communication. Direct patient communication has been proposed previously by eminent radiological authors such as Berlin, the chief commentator on the topics of error and malpractice in clinical radiology [1], who posed the question "Communicating Results of All Radiologic Examinations Directly to Patients: Has the Time Come?" in an editorial twelve years ago. This practice, however, has never taken widespread hold internationally.

Notably, radiological screening programmes have always been an exception to this referrer-led communication pattern, having been early adopters of direct communication. Breast screening programmes usually communicate client test results directly to patients. Over twenty years ago, a survey of 400 patients regarding breast screening in the USA [2] where results were sent directly to patients concluded that patients preferred a double-read delayed report with the results sent directly by post rather than an immediate single-read report

Julie Cox

julie.cox@chsft.nhs.uk

1 Department of Radiology, City Hospitals Sunderland NHS Foundation Trust, Kayll Road, Sunderland, Tyne and Wear SR4 7TP, UK

2 Faculty of Health Sciences and Wellbeing, University of Sunderland, Sciences Complex, Sunderland, Tyne and Wear SR1 3SD, UK by a radiologist on the same day as their mammograms. It is interesting to note that the participants appear to have understood the benefits of the additional quality assurance that a second review of images would bring, valuing this over an immediate result. It is unknown whether the findings would apply today, with society's current level of demand for immediate and instant (although often incorrect) answers.

The language employed in written clinical communication is vitally important, especially when conveyed to a patient. An American survey [3] analysed 43 different template letters for the communication of mammography results were available from the American College of Radiologists and two transcription services. Using two linguistic indices to analyse the letters, the authors concluded that the design and scope of the letters made them difficult to understand, in a manner that was incomprehensible to half the US population of women. The findings were consistent with those of other authors in that much patientcentred material internationally is written in styles that are discordant with reading levels of the general public. The authors make the salient point that if radiology results are to be conveyed to patients, the information should be presented in a form that patients can understand. The discipline of radiology is pressed for time, with a noted workforce shortage. Many radiologists juggling competing demands may be resistant to the notion of producing alternative "patient-focused" reports. If direct radiology communication with patients is to be adopted, then, alternative plain language reports will be required.

Currently, it is unknown how many patients may wish to receive results directly from a radiology department. It could be argued that this places a false demand on radiology which is already under siege from increasing workloads. In an Italian cross-sectional study involving 1171 patients attending for 
outpatient CT, ultrasound, or MRI examinations, $45 \%$ requested information about the results of the examination [4]. There were no significant differences regarding gender or anxiety beforehand, or if the study was the first or a follow-up examination. Older patients enquired less frequently than younger ones, but the differences were not statistically significant. Educational levels were highly significant, with $36.4 \%$ of patients with an elementary school education asking for the results, compared with $41.5 \%$ with a middle school education and $55.1 \%$ with a high school education or university degree $(p<0.001)$.

An alternative method of passive communication with patients will undoubtedly develop through the advent of widespread highly integrated electronic records with patient portals, where the patient's record as a whole (including test results) is visible for perusal. Whether patients fully realise the extent of their personal data available through online accessible medical records is not well understood. Patients do appear amenable to receiving their results whatever the content based on a study presenting simulated scenarios [5]. Most patients wanted the reports regardless of the findings, with more than $60 \%$ wanting immediate access to their radiology reports when the findings were normal. About one-half $(47.2 \%)$ said they wanted immediate access if the findings were seriously abnormal, and $45 \%$ said they wanted immediate access if the findings were inconclusive.

Traditionally in the radiology literature, communication with patients is generally defined as conveying the result of the radiological examination. Communication could also be construed as a much broader concept than the transmission of a few lines of a report. This raises the vexed and challenging topic of radiological discrepancy and error. Communication with patients about radiological errors and revised or appended diagnoses (possibly amended with additional clinical information) remains topical and relevant. A 2005 editorial [6] comments, with some validity, if patients have ownership of their test results from direct communication with radiologists, abnormal results are less likely to be overlooked. This knowledge potentially improves safety and patient satisfaction with services, as patients are co-customers along with referrers. Gutzeit et al [7] report a study in which patients given an opportunity to discuss MRI results personally with a radiologist after the scan perceived the opportunity to discuss their imaging findings with a radiologist to be a characteristic of a good radiology consultation. A greater number of patients in the direct communication group experienced significantly higher bonding and only wanted in the future to be examined in the department with communication. The concept of patient "bonding" with radiology departments is indeed intriguing. Certainly in this study did lead to the perception of higher levels of competence in the radiology service provided, but two challenges are immediately evident, while providing personal consultation in private practice may be feasible, in public hospitals with high volumes of examinations, this is clearly impractical in terms of service delivery. Equally, providing rapid verbal "provisional-type" reports for patients in this setting may result in confusion if reports are amended or altered in their final version. High and increasing levels of use of outsourced teleradiology services are viewed by clinicians with some distrust compared with local-provided radiology services [8]. This view may also filter through to patients should the specific origin of the report be known to them.

A recent commentary by Maskell [9] asserts that although radiologists know that a level of discrepancy is normal and expected within an opinion-based specialty, both clinicians and patients have unrealistic expectations of the accuracy of radiological interpretation. Many patients still regard the discovery of a radiological discrepancy or missed diagnosis with hindsight (an everyday occurrence in clinical practice) as a "shocking and exceptional" event. Good quality communication through education and dialogue with patients and colleagues about error and the limitations of imaging would appear to be the only answer to misperceptions about radiological error within medicine and in the wider community.

Funding The authors state that this work has not received any funding.

\section{Compliance with ethical standards}

Guarantor The scientific guarantor of this publication is Dr. J Cox. This is an editorial and the opinions represent the view of Dr. Cox and Dr. Graham and not their employers.

Conflict of interest The authors of this manuscript declare no relationships with any companies, whose products or services may be related to the subject matter of the article.

Statistics and biometry No complex statistical methods were necessary for this paper.

Informed consent This is an editorial and informed consent is not applicable.

Ethical approval Institutional Review Board approval was not required because this is an editorial.

Methodology

- Editorial

\section{References}

1. Berlin L (2007) Communicating results of all radiologic examinations directly to patients: has the time come? AJR Am J Roentgenol 189(6):1275-1282

2. Hulka CA, Slanetz PJ, Halpern EF et al (1997) Patients' opinion of mammography screening services: immediate results versus delayed results due to interpretation by two observers. AJR Am J Roentgenol 168(4):1085-1089 
3. Marcus EN, Koru-Sengul T, Miao F, Yepes M, Sanders L (2014) How do breast imaging centers communicate results to women with limited English proficiency and other barriers to care? J Immigr Minor Health 16(3):401-408

4. Capaccio E, Podestà A, Morcaldi D, Sormani MP, Derchi LE (2010) How often do patients ask for the results of their radiological studies? Insights Imaging 1(2):83-85

5. Johnson A, Easterling D, Nelson R, Chen M, Frankel RM (2012) Access to radiologic reports via a patient portal: clinical simulations to investigate patient preferences. J Am Coll Radiol 9(4):256-263

6. Johnson A, Hawkins H, Applegate K (2005) Web-based results distribution: new channels of communication from radiologists to patients. J Am Coll Radiol 2(2):168-173
7. Gutzeit A, Heiland R, Sudarski S et al (2019) Direct communication between radiologists and patients following imaging examinations. Should radiologists rethink their patient care? Eur Radiol 29(1):224 231

8. Graham Y, Hayes C, Mehrotra P, Spratt J, Siddle K, Cox J (2019) Clinicians' perceptions of the quality of outsourced radiology and actions taken around perceived imaging errors in practice. Eur Radiol 29(4):1649-1654

9. Maskell G (2019) Error in radiology-where are we now? Br J Radiol 92:20180845

Publisher's note Springer Nature remains neutral with regard to jurisdictional claims in published maps and institutional affiliations. 